MATHEMATICS OF COMPUTATION

Volume 67, Number 221, January 1998, Pages 353-356

S 0025-5718(98)00887-4

\title{
EVALUATION OF DISCRETE LOGARITHMS IN A GROUP OF $p$-TORSION POINTS OF AN ELLIPTIC CURVE IN CHARACTERISTIC $p$
}

\author{
I. A. SEMAEV
}

\begin{abstract}
We show that to solve the discrete log problem in a subgroup of order $p$ of an elliptic curve over the finite field of characteristic $p$ one needs $O(\ln p)$ operations in this field.
\end{abstract}

Let $F_{q}$ be the finite field of $q=p^{l}$ elements. We define an elliptic curve $E$ over $F_{q}$ to be an equation of the form

$$
y^{2}=x^{3}+A x+B .
$$

We suppose $p \neq 2,3$. Let $E\left(F_{q}\right)$ be the set of points $E$ rational over $F_{q}$. It is known that $\left|N_{q}-q-1\right| \leq 2 q^{1 / 2}$ with $N_{q}=\left|E\left(F_{q}\right)\right|$. The set $E\left(F_{q}\right)$ is a finite abelian group with the "infinite point" $P_{\infty}$ as the identity element.

The discrete logarithm problem is to compute an integer $n$ such that $Q=n P$, where $Q, P \in E\left(F_{q}\right)$, if such an $n$ exists. This problem is of great significance in cryptology [1], [2]. Suppose that the point $P$ generates a subgroup $\langle P\rangle$ of order $m$. If $(m, p)=1$, then the subgroup $\langle P\rangle$ is isomorphic to some multiplicative subgroup of an extension $F_{q^{k}}$ where $q^{k} \equiv 1(\bmod m)$. The values of the isomorphism from $\langle P\rangle$ to $F_{q}^{*}$ can be evaluated in a very simple manner. The complexity of the algorithm is no more than $O(\ln m)$ operations in $F_{q^{k}}$ [3], [4], [5]. Thus when $k$ is small we have an algorithm for the discrete log problem in $\langle P\rangle$ more effective than the algorithms of the kind shown in [6], [7]. However if $(m, p) \neq 1$ the reduction above is impossible. We have $m=p^{s} m_{1}$ where $s>0$ and $\left(m_{1}, p\right)=1$. Consequently, the discrete log problem in $\langle P\rangle$ is reduced to a discrete log problem in subgroups of order $m_{1}$ and $p$. For the subgroup of order $m_{1}$ one can apply the reduction to a multiplicative subgroup of the extension $F_{q^{k}}$ with minimal $k$ such that $q^{k} \equiv 1\left(\bmod m_{1}\right)$.

In this paper we construct an isomorphism from the subgroup of order $p$ to the additive group of $F_{q}$. One can evaluate the values of this isomorphism with $O(\ln p)$ operations in $F_{q}$. Thus the discrete log problem in a subgroup of order $p$ of an elliptic curve over the field of characteristic $p$ is polynomial.

Assume that a point $P \in E\left(F_{q}\right)$ generates a subgroup of order $p$. We let $t_{R}$ denote a local parameter at a point $R$ the coordinates of which are $\left(x_{R}, y_{R}\right)$ if $R \neq P_{\infty}$. If $R$ is not of order 2 or $P_{\infty}$, then $t_{R}=x-x_{R}$. If $R \neq P_{\infty}$ is a point of order 2 , then $t_{R}=y$. Finally $t_{P_{\infty}}=x / y$. It must be noted that a point $R$ of order

Received by the editor August 30, 1995 and, in revised form, September 11, 1996.

1991 Mathematics Subject Classification. Primary 94A60.

Key words and phrases. Cryptography, elliptic curves, discrete logarithms.

(C)1998 American Mathematical Society 
2 on $E$ has the coordinates $\left(x_{R}, 0\right)$. Let us take up to the end of this article a point $R \in\langle P\rangle-P_{\infty}$.

It is known that $E$ is isomorphic to the quotient of the group of divisors of degree 0 by the subgroup of principal divisors, a point $Q$ corresponding to a divisor $D_{q}=\sum n_{T} T$ where $Q$ is a sum on $E$ of the points $T$ taken with multiplicities $n_{T}$. For example, $D_{Q}=(Q)-\left(P_{\infty}\right)$. If $Q \in\langle P\rangle$, then $p D_{Q}$ is a principal divisor that is denoted $\left(f_{Q}\right)=p D_{Q}$ for some function $f_{Q}$ on $E$.

Lemma 1. Let $f$ be a function on $E$ such that $(f)=p D$ for some nonprincipal divisor $D$. Let $f^{\prime}=d f / d x$ be the derivative of $f$ with respect to $x$. Then $\left(f^{\prime}\right)=$ $(f)-(y)$.

Proof. Let $v_{Q}$ be the valuation at the point $Q$. Let $D=\sum n_{Q} Q$. Set $f=t_{Q}^{p l_{Q}} f_{1}$ where $f_{1}$ is regular at $Q$ and $f_{1}(Q) \neq 0$. First we assume that $Q$ is not in the divisor of the function $y$; that is, $Q$ is neither of order 2 nor $P_{\infty}$. Hence $d f / d x=$ $d f / d\left(x-x_{Q}\right)=t_{Q}^{p l_{Q}} d f_{1} / d t_{Q}$. The function $d f_{1} / d t_{Q}$ is regular at $Q$ [8]. Then $v_{Q}\left(f^{\prime}\right)=p l_{Q}+m_{Q}$ where $m_{Q}=v_{Q}\left(d f_{1} / d t_{Q}\right) \geq 0$. Let $Q$ be a point of order 2 . Then

$$
d f / d x=(d f / d y) d y / d x=y^{p l_{Q}}\left(\left(3 x^{2}+A\right) / 2 y\right) d f_{1} / d y,
$$

where $d y / d x=\left(3 x^{2}+A\right) / 2 y$. Since $v_{Q}\left(\left(3 x^{2}+A\right) / 2 y\right)=-1$, in this case $v_{Q}\left(f^{\prime}\right)=$ $p l_{Q}+m_{Q}-1$, with $m_{Q}=v_{Q}\left(d f_{1} / d t_{Q}\right) \geq 0$. Set $Q=P_{\infty}$. Then

$$
d f / d x=(d f / d(x / y)) d(x / y) / d x=(x / y)^{p l_{Q}}\left(\left(-x^{3}+A x+B\right) / 2 y^{3}\right) d f_{1} / d(x / y),
$$

where $d(x / y) / d x=\left(-x^{3}+A x+B\right) / 2 y^{3}$. Hence we have $v_{Q}\left(f^{\prime}\right)=p l_{Q}+m_{Q}+3$ because $v_{P_{\infty}}\left(\left(-x^{3}+A x+B\right) / 2 y^{3}\right)=3$ and $m_{Q}=v_{Q}\left(d f_{1} / d t_{Q}\right) \geq 0$. Let $D_{1}=$ $\sum m_{Q} Q$. As we have seen $D_{1}$ is a positive divisor. On the other hand, since $\left(f^{\prime}\right)=(f)-(y)+D_{1}$, the divisor $D_{1}$ is principal. So $D_{1}=0$ and the lemma is proved.

Consider the following map $\phi$ of points of the group $\langle P\rangle$ to $F_{q}$ :

$$
\phi(Q)=\left(f_{Q}^{\prime} / f_{Q}\right)(R), \quad \phi\left(P_{\infty}\right)=0 .
$$

Lemma 2. The value $\phi(Q)$ is well defined. The map $\phi$ is an isomorphic embedding of $\langle P\rangle$ into the additive group of $F_{q}$.

Proof. Let $D_{Q}^{\prime}, D_{Q}$ be linearly equivalent divisors. Hence there is the function $g$ such that $(g)=D_{Q}-D_{Q}^{\prime}$. So if $(f)=p D_{Q}^{\prime}$, then $g^{p} f=f_{Q}$. It is easy to see that $f_{Q}^{\prime} / f_{Q}=f^{\prime} / f$ so that $\phi(Q)$ is well defined. One can always take $D_{Q}$ rational over $F_{q}$. So $f_{Q}^{\prime} / f_{Q}(R) \in F_{q}$, since $R$ is rational over $F_{q}$. Let us show that $\phi$ is a homomorphism. Let $Q_{i} \in\langle P\rangle$ and $\left(f_{Q_{i}}\right)=p D_{Q_{i}}, i=1,2$. Define $D_{Q_{1}+Q_{2}}=D_{Q_{1}}+D_{Q_{2}}$. Then

$$
\left(f_{Q_{1}+Q_{2}}\right)=p D_{Q_{1}+Q_{2}}=\left(f_{Q_{1}} f_{Q_{2}}\right) .
$$

So the functions $f_{Q_{1}+Q_{2}}$ and $f_{Q_{1}} f_{Q_{2}}$ are equal up to a multiplicative constant. Hence

$$
f_{Q_{1}+Q_{2}}^{\prime} / f_{Q_{1}+Q_{2}}=f_{Q_{1}}^{\prime} / f_{Q_{1}}+f_{Q_{2}}^{\prime} / f_{Q_{2}}
$$

We have proved that $\phi$ is a homomorphism. Since $\phi$ is non-vanishing on $\langle P\rangle$, then $\phi$ is an isomorphism and the lemma is proved. 
The construction of this isomorphism can also be derived from a general result of Serre [9, pp. 40-41].

Lemma 3. Let $Q \in\langle P\rangle$. Then the value of the function $f_{Q}^{\prime} / f_{Q}$ at $R$ can be evaluated with $O(\ln p)$ operations in $F_{q}$.

Proof. Let us take $D_{Q}=(Q+S)-(S)$ where $S$ is of order 2 exactly. Denote by $\psi_{k}$ the function such that

$$
\left(\psi_{k}\right)=k(Q+S)-(k Q+S)-(k-1)(S) .
$$

Clearly $\psi_{p}=f_{Q}$ up to a multiplicative constant. Let $k=k_{1}+k_{2}, k_{i} \geq 0$. Then the following identity is valid [4]:

$$
\psi_{k} \lambda_{k_{1}, k_{2}}=\psi_{k_{1}} \psi_{k_{2}}
$$

where $\lambda_{k_{1}, k_{2}}$ is a function such that

$$
\left(\lambda_{k_{1}, k_{2}}\right)=(k Q+S)-\left(k_{1} Q+S\right)-\left(k_{2} Q+S\right)+(S) .
$$

The identity (1) gives us a method for evaluation of the value $f_{Q}^{\prime} / f_{Q}(R)$. Indeed, from (1) we have

$$
\psi_{k}^{\prime} / \psi_{k}=\psi_{k_{1}}^{\prime} / \psi_{k_{1}}+\psi_{k_{2}}^{\prime} / \psi_{k_{2}}-\lambda_{k_{1}, k_{2}}^{\prime} / \lambda_{k_{1}, k_{2}} .
$$

Hence the function $\psi_{k}^{\prime} / \psi_{k}$ is expressed by a linear combination of $O(\ln k)$ functions of the form $\lambda_{k_{1}, k_{2}}^{\prime} / \lambda_{k_{1}, k_{2}}$. Let $\eta_{k_{1}, k_{2}}$ be

$$
\left(\eta_{k_{1}, k_{2}}\right)=\left(\left(k_{1}+k_{2}\right) Q+S\right)+\left(-k_{1} Q+S\right)+\left(-k_{2} Q+S\right)-3(S),
$$

$\kappa_{k}$ be

$$
\left(\kappa_{k}\right)=(k Q+S)+(-k Q+S)-2(S) .
$$

Let us note that $\eta_{k_{1}, k_{2}}(X-S), \kappa_{k_{1}}(X-S)$ are linear functions in $x, y$. The coefficients of these functions are determined by the coordinates of the points $\left(k_{1}+k_{2}\right) Q, k_{1} Q, k_{2} Q$. We have the equality

$$
\lambda_{k_{1}, k_{2}}=\eta_{k_{1}, k_{2}} \kappa_{k_{1}}^{-1} \kappa_{k_{2}}^{-1} .
$$

Then it is easy to see that

$$
\lambda_{k_{1}, k_{2}}^{\prime} / \lambda_{k_{1}, k_{2}}=\eta_{k_{1}, k_{2}}^{\prime} / \eta_{k_{1}, k_{2}}-\kappa_{k_{1}}^{\prime} / \kappa_{k_{1}}-\kappa_{k_{2}}^{\prime} / \kappa_{k_{2}} .
$$

The functions on the right-hand side of this equality can be determined from the following considerations. Let $\delta=a x+b y+c$ be any linear function in $x, y$. Let $\delta_{1}=\delta(X+S)$. We have to find the value of the function $\delta_{1}^{\prime} / \delta_{1}$ at some point $R$. Express this function by the functions $\delta, \delta^{\prime}$, where $\delta^{\prime}=d \delta / d x=a+b\left(3 x^{2}+A\right) / 2 y$. We have $d \delta=\left(2 y \delta^{\prime}\right) d x / 2 y$. It is known [8] that $d x / 2 y$ is an invariant differential on $E$. In other words $(d x / 2 y)(X+S)=(d x / 2 y)(X)$ for any point $S \in E$. So denoting $\delta_{2}=2 y \delta^{\prime}$ we have $d \delta(X+S)=\delta_{2}(X+S) d x / 2 y$. Hence $\delta_{1}^{\prime}=\delta_{2}(X+S) / 2 y$. Finally,

$$
\delta_{1}^{\prime} / \delta_{1}=\delta_{2}(X+S) / 2 y \delta(X+S) .
$$

Thus we have to evaluate the values of $O(\ln k)$ functions of type $\delta^{\prime} / \delta$ where the coefficients are determined by the coordinates of the points $\left(k_{1}+k_{2}\right) Q, k_{1} Q, k_{2} Q$. Altogether we have to evaluate $O(\ln k)$ such points. Since the points of this set are expressed by the same set, the complexity of this calculation is no more than $O(\ln k)$ operations in $F_{q}$.

From (2) it follows that the functions $\eta_{k_{1}, k_{2}}^{\prime} / \eta_{k_{1}, k_{2}}, \kappa_{k_{i}}^{\prime} / \kappa_{k_{i}}$ are regular at $R$. Thus the total complexity of evaluation of the values of the functions $\psi_{k}^{\prime} / \psi_{k}$ at $R$ 
takes no more than $O(\ln k)$ operations in $F_{q}$. Note that the calculations above are performed in the extension of $F_{q}$ obtained by adjoining the point of order 2. Since this extension has degree at most 3 , the complexity of the operations in this field is proportional to those in $F_{q}$. This proves the lemma.

From Lemma 3 it follows that the complexity of the discrete log problem in the group $\langle P\rangle$ is no more than $O(\ln p)$ operations in $F_{q}$. Actually, to get an integer $n$ such that $Q=n P$ in $E\left(F_{q}\right)$ one must evaluate the values $\phi(Q), \psi(P) \in F_{q}$, then $n=\phi(Q)(\phi(P))^{-1}$.

In [10] H.-G. Ruck generalizes the results of the present paper to curves of arbitrary genus.

\section{REFERENCES}

1. V. Miller, Use of elliptic curves in cryptography, Advances in Cryptology-Crypto '85, Springer-Verlag, New York, 1986, 417-426. MR 88b:68040

2. N. Koblitz, Elliptic curve cryptosystems, Math. Comp. 48 (1987), 203-209. MR 88b:94017

3. A. Menezes, S. Vanstone, and O. Tatsuaki, Reducing elliptic curve logarithms to logarithms in a finite field, Proc. 23rd ACM Sympos. Theory of Computing, 1991, pp. 80-89.

4. И. А. Семаев, Быстрый алгоритм вычисления спаривания А. Вейля на әллиптической кривой, International Conference "Modern Problems in Number Theory", Russia, Tula, Sept. 20-25, 1993, Abstracts of papers.

5. G. Frey and H.-G. Ruck, A remark concerning m-divisibility and the discrete logarithm in the divisor class group of curves, Math. Comp. 62 (1994), 865-874. MR 94h:11056

6. S. Pohlig and M. Hellman, An improved algorithm for computing logarithms over GF $(p)$ and its cryptographic significance, IEEE Trans. Inform. Theory, IT-24 (1978), 106-110. MR $\mathbf{5 8 : 4 6 1 7}$

7. J. M. Pollard, Monte-Carlo methods for index computation $(\bmod p)$, Math. Comp. 32 (1978), 918-924. MR 58:10684

8. J. H. Silverman, The arithmetic of elliptic curves, Springer-Verlag, 1986. MR 87g:11070

9. J. P. Serre, Sur la topologie des variétés algébriques en caractéristique p, Sympos. Internac. Topologia Algebraica, Mexico City, 1956, 24-53. MR 20:4559

10. H.-G. Ruck, A remark on the paper "Evaluation of discrete logarithms on some elliptic curves, by I. A. Semaev", communication to "Mathematics of Computation".

43-2 Profsoyusnaya ul., Apt. 723, 117420 Moscow, Russia 